\title{
Consumo de cafeína: o que aconselhar na preconceção e gravidez?
}

Filipa Matias, ${ }^{1-2}$ Alice Jeri, ${ }^{1-3}$ Sofia Rodrigues ${ }^{1-2}$

\section{RESUMO}

Objetivo: Rever a evidência sobre a associação entre consumo de cafeína em mulheres saudáveis - no período preconceção ou durante a gravidez - e o risco de abortamento espontâneo ou nado morto.

Fontes de dados: Bases de dados MEDLINE, National Guideline Clearinghouse, Canadian Medical Association Practice Guidelines Infobase, Cochrane Library, Bandolier, DARE, Trip Datebase e Índex de Revistas Médicas Portuguesas.

Métodos de revisão: Revisão baseada na evidência de artigos publicados nos últimos cinco anos (de 1 de agosto de 2010 a 31 de agosto de 2015), nas línguas inglesa, francesa, italiana, espanhola e portuguesa. Foram utilizados os termos MESH 'caffeine', 'spontaneous abortion' e 'fetal death'. Para avaliação do nível de evidência (NE) e força de recomendação foi utilizada a escala Strength of recommendation taxonomy, da American Family Physician.

Resultados: Foram obtidos 84 artigos, dos quais quatro cumpriam os critérios de inclusão: três revisões sistemáticas com meta-análises (todas com NE 2) e um estudo coorte prospetivo (NE 1). Parece existir um aumento do risco de abortamento espontâneo ou nado morto com o consumo moderado a elevado de cafeína (superior a 100-150mg/dia) durante a gravidez. Não há evidência suficiente que demostre associação de risco no consumo durante a preconceção.

Conclusões: $O$ consumo moderado a elevado de cafeína durante a gravidez está associado ao aumento do risco de abortamento espontâneo e de nado morto (força de recomendação B), aconselhando-se um consumo nulo ou mínimo de cafeína (<100-150mg/dia) durante este período. Não há ainda evidência suficiente que demostre associação entre consumo durante a preconceção e a morte in utero. São necessários novos estudos que reforcem a evidência e definam as doses seguras de consumo de cafeína pela mulher nestes períodos.

Palavras-chave: Cafeína; Abortamento espontâneo; Morte fetal; Nado morto; Gravidez; Preconceção

\section{INTRODUÇÃO}

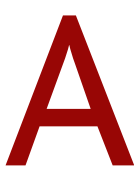
cafeína é uma substância psicoativa, estimulante do sistema nervoso central. Está presente em múltiplos alimentos e bebidas como o café, chá, chocolate, refrigerantes, bebidas energéticas, entre outros. O café, uma das bebidas mais consumidas a nível mundial, é a bebida mais frequentemente associada à cafeína. ${ }^{1}$ Em Portugal, o seu consumo ronda os $4,7 \mathrm{~kg} /$ pessoa/ano, segundo os dados da European Coffee Federation, ${ }^{2}$ prevendo-se até 2021 um aumento deste valor, sobretudo no sexo feminino. ${ }^{3}$

O consumo da cafeína pode ter impacto na progressão da gravidez, uma vez que a substância atravessa a barreira placentária e pode ser detetada no fluído amniótico. O feto apresenta reduzida capacidade de metabolização da cafeína, a qual prejudica o desenvolvimento celular do embrião ou

1. Médicas Internas de Medicina Geral e Familiar

2. USF Novo Sentido, ACeS Porto Oriental

3. USF Barão de Nova Sintra, ACeS Porto Oriental feto. ${ }^{1}$ Tal facto é também potenciado pela diminuição da eliminação hepática da cafeína que ocorre durante a gravidez.

O mecanismo através do qual a cafeína compromete o crescimento fetal ainda permanece incerto. Tem sido postulada a hipótese de que aumente a libertação de catecolaminas, levando à vasoconstrição na circulação uteroplacentária e consequente hipoxia fetal, condicionando o seu crescimento e desenvolvimento. ${ }^{4}$ Com efeito, foi documentada uma diminuição de $25 \%$ no fluxo placentário interviloso após a ingestão materna de apenas $200 \mathrm{mg}$ de cafeína. ${ }^{5}$ Outra hipótese é a de que a cafeína aumente a concentração intracelular de adenosina monofosfato cíclico através da inibição da fosfodiesterase, influenciando a divi-são celular e afetando, desta forma, o crescimento fetal. ${ }^{6-7}$

Possíveis efeitos adversos gestacionais do consumo da cafeína incluem o abortamento espontâneo (antes das 22 semanas) e o nado morto (durante e após as 22 semanas) ${ }^{8.9}$ Estas ocorrências são frequentes na população em geral, tendo a busca de fatores etiológicos ambientais motivado 
extensas pesquisas nem sempre conclusivas. ${ }^{10}$ Outros possíveis efeitos adversos do consumo da cafeína durante a gravidez incluem a diminuição no crescimento intrauterino, a redução do peso ao nascer e o risco de teratogénese.

Em contraponto, sabe-se que os sintomas associados à gravidez (como aversão a paladares e odores, náuseas e vómitos) são mais comuns em gestações saudáveis que resultarão em nados-vivos, sendo menos frequentes naquelas que terminarão em abortamento espontâneo. ${ }^{11}$ Esta relação é atribuída a um pregnancy signal (concentração de hormonas gravídicas) mais forte, um sinal associado a uma gestação viável. Foi demonstrado que o consumo de cafeína tende a diminuir com o agravamento dos sintomas associados à gravidez durante as primeiras semanas, pelo que o menor consumo poderia ser consequência da viabilidade da gestação (elevado pregnancy signal), ao invés de um elevado consumo ser causador de abortamento (efeito de causalidade inversa). Estes sintomas podem, portanto, constituir-se como confundidores importantes nos estudos.

Outro fator de difícil ajuste é o tabagismo, frequentemente associado ao consumo de café e com riscos próprios para a gestação, alterando adicionalmente a metabolização da cafeína. ${ }^{12}$

As recomendações atuais relativamente à quantidade permitida de consumo de cafeína durante a gravidez são díspares, aconselhando-se geralmente ingestão diária inferior a 200-300mg/dia. ${ }^{13-14}$ As quantidades médias aproximadas de cafeína doseada em bebidas e alimentos mais comuns estão descritas no Quadro I.

O presente trabalho tem como objetivo rever a evidência sobre a associação entre consumo de cafeína em mulheres saudáveis - no período preconceção ou durante a gravidez e o risco de abortamento espontâneo ou nado morto.

\section{MÉTODO}

Realizou-se uma pesquisa bibliográfica de normas de orientação clínica (NOC), revisões sistemáticas, meta-análises, estudos originais nas bases de dados MEDLINE, $\mathrm{Na}$ tional Guideline Clearinghouse, Canadian Medical Association Practice Guidelines Infobase, Cochrane Library, Bandolier, DARE, Trip Datebase e Índex de Revistas Médicas Portuguesas de artigos publicados nos últimos cinco anos, de 1 de agosto de 2010 a 31 de agosto de 2015, nas línguas inglesa, francesa, italiana, espanhola e portuguesa. Foram utilizados os termos MESH 'caffeine', 'spontaneous abortion' $\mathrm{e}$ 'fetal death'. Foram também utilizados os termos em português “cafeína”, “abortamento espontâneo" e "morte fetal” na pesquisa no Índex de Revistas Médicas Portuguesas. A pes-

\begin{tabular}{|c|c|c|}
\hline \multicolumn{3}{|c|}{$\begin{array}{l}\text { QUADRO I. Quantidades aproximadas de cafeína em } \\
\text { alimentos comuns (adaptação) }\end{array}$} \\
\hline BEBIDAS & Dose/porção & mg de cafeína \\
\hline \multicolumn{3}{|l|}{ Café } \\
\hline De saco & 200mL (1 chávena) & 90 \\
\hline Instantâneo & 237mL (1 chávena) & $76-106$ \\
\hline Descafeínado & 237mL (1 chávena) & $5-15$ \\
\hline Expresso & $60 \mathrm{~mL}$ & 80 \\
\hline \multicolumn{3}{|l|}{ Chá } \\
\hline Preto & 220mL (1 chávena) & 50 \\
\hline Verde & 237mL (1 chávena) & $24-45$ \\
\hline Coca-cola & $355 \mathrm{~mL}$ (1 lata) & 40 \\
\hline Chocolate de leite & $28 g$ (4 quadrados) & 7 \\
\hline
\end{tabular}

quisa bibliográfica foi feita no dia 1 de setembro de 2015.

Definiram-se como critérios de inclusão:

- População: mulheres saudáveis em período de preconceção e/ou grávidas;

- Intervenção: consumo de produtos com cafeína em doses variáveis;

- Comparação: ausência, ou consumo mínimo (<100150mg/dia), de produtos com cafeína;

- Outcome: ocorrência de casos de abortamento espontâneo ou nado morto.

Foram excluídas mulheres com antecedentes patológicos ou fatores de risco conhecidos, em tratamento de fertilidade, com história de abortamentos de repetição ou com consumo concomitante de outras substâncias potencialmente tóxicas, com exceção do tabaco.

Para avaliação do nível de evidência (NE) e força de recomendação (FR) foi utilizada a escala Strength of recommendation taxonomy (SORT), ${ }^{15}$ da American Family Physician (Quadro II). Para avaliação da qualidade metodológica dos artigos foram utilizados os critérios de Downs e Black e aplicado o Appraisal of Guidelines Research \& Evaluation Instrument (AGREE) na avaliação das NOCs. ${ }^{16-18}$

\section{RESULTADOS}

A pesquisa inicial identificou 84 artigos, dos quais se excluíram cinco por se encontrarem duplicados e 75 por não se enquadrarem no objetivo da revisão, por não cumprirem critérios de inclusão ou por não revelarem qualidade metodológica (essencialmente por pouca qualidade de informação e validade interna). Após a aplicação do AGREE, os autores não recomendam a inclusão da NOC encontrada, 


\begin{tabular}{|c|c|c|}
\hline \multicolumn{3}{|r|}{ Strength of Recommendation Taxonomy, da American Family Physician } \\
\hline & Classificação & Significado \\
\hline FR & $\begin{array}{l}\text { A } \\
B \\
\text { C }\end{array}$ & $\begin{array}{l}\text { Recomendação baseada em evidência consistente, de boa qualidade, orientada para o doente. } \\
\text { Recomendação baseada em evidência inconsistente, de qualidade limitada, orientada para o doente. } \\
\text { Recomendação baseada em consensos, prática clínica, opinião, orientada para a doença ou séries de casos } \\
\text { acerca do diagnóstico, tratamento prevenção ou rastreio. }\end{array}$ \\
\hline NE & $\begin{array}{l}1 \\
2 \\
3\end{array}$ & $\begin{array}{l}\text { Estudos de boa qualidade, evidência orientada para o doente. } \\
\text { Estudos de qualidade limitada, evidência orientada para o doente. } \\
\text { Outra evidência. }\end{array}$ \\
\hline
\end{tabular}

Legenda: FR - Força de recomendação; NE - Nível de evidência.

particularmente devido à baixa pontuação auferida nos domínios de rigor de desenvolvimento, clareza da apresentação e aplicabilidade. Foram incluídos quatro artigos: três revisões sistemáticas com meta-análise e um estudo de coorte prospetivo. O fluxograma da seleção dos estudos encontra-se explanado na Figura 1.

A revisão sistemática com meta análise elaborada por Li e colaboradores, em 2015, englobou 26 estudos: 13 estudos caso-controlo e 13 estudos de coorte. Destes, apenas 20 abordavam o consumo de cafeína. Foi utilizada uma análise quantitativa para detetar a eventual associação entre o consumo de cafeína durante a gestação e a perda da gravidez (através de questionários, entrevistas e registos clínicos), explorando uma relação dose-resposta. Nesta análise foi considerada exposição ligeira à cafeína como consumo inferior a 150mg/dia; exposição moderada entre 150 e 300mg/dia; e exposição elevada quando consumo igual ou superior a $301 \mathrm{mg} /$ dia. Os limites temporais para o abortamento espontâneo abrangeram o período antes das 20 semanas, sendo a não-evolução posterior da gravidez considerada como nado morto.

Globalmente, o consumo de cafeína associou-se ao aumento do risco de abortamento espontâneo ou nado morto - Odds Ratio (OR) 1,47 ( $p<0,01$; intervalo de confiança [IC] 95\% 1,31-1,66; heterogeneidade $\left.\left[\mathrm{I}^{2}\right]=70,9 \%\right)$. Foi encontrado um efeito dose-resposta a partir do consumo de 149,5mg/dia, não se estabelecendo associação para a exposição ligeira. Também se verificou um aumento no risco de $19 \%$ para cada aumento de $150 \mathrm{mg} /$ dia no consumo de cafeína - OR 1,19 (IC 95\% 1,16-1,23). Apesar de se encontrar marcada heterogeneidade entre os estudos na primeira análise, esta deixou de ser significativa após a exclusão de dois estudos específicos $\left(\mathrm{I}^{2}=48 \%\right)$, mantendo-se a asso- ciação. De referir que os autores apontaram como limitação desta revisão um possível viés de publicação (pesquisa realizada apenas na MEDLINE) (NE 2) (Quadro III).

Na revisão sistemática com meta-análise de Greenwood e colaboradores, de 2014, foram incluídos 53 estudos observacionais. Com relevância para os outcomes deste trabalho foram considerados, relativamente ao abortamento espontâneo, 26 estudos (14 estudos de coorte e 12 casoscontrolo); e, relativamente ao nado morto, cinco estudos (três estudos de coorte e dois casos-controlo). Nesta análise foi estimado o consumo de cafeína através da conversão dos produtos em cafeína, atribuindo 100mg de cafeína por unidade de café e $60 \mathrm{mg}$ de cafeína por unidade de outras fontes (chá ou coca-cola®).

No que concerne ao abortamento espontâneo (definido neste estudo como morte in utero antes das 24 semanas de gestação), a meta-análise dos dados encontrou um risco relativo (RR) de 1,14 a partir do consumo de $100 \mathrm{mg} /$ dia de cafeína, com uma relação dose-resposta linear ( $p<0,001$; IC 95\% 1,10-1,19, $\mathrm{I}^{2}=89 \%$ ). Dada a elevada heterogeneidade verificada foi excluído o estudo com maior impacto na variabilidade, o que resultou na redução da heterogeneidade (RR 1,11; IC 95\% 1,07-1,14; $\mathrm{I}^{2}<44 \%$ ), mantendo-se a relação inicialmente encontrada. Assim, demonstrou-se um aumento discreto, mas consistente, da incidência de abortamento espontâneo com o aumento do consumo diário de cafeína.

Relativamente ao nado morto (definido como morte in uteroapós as 24 semanas de gestação), a meta-análise dos dados encontrou um RR de 1,19 a partir do consumo de $100 \mathrm{mg} /$ dia de cafeína, com relação dose-resposta linear ( $p=0,007$; IC 95\% 1,05-1,35, $\mathrm{I}^{2}=82 \%$ ). Também aqui se verificou elevada heterogeneidade, tendo sido reduzida após es- 
tratificação de um estudo que apenas considerou o consumo de café como fonte de cafeína $\left(\mathrm{I}^{2}<50 \%\right)$, mantendo-se a relação inicialmente encontrada. Foi possível demonstrar um aumento discreto, mas consistente, da incidência de nado morto com o aumento do consumo diário de cafeína.

Como conclusão, esta revisão sistemática aconselha que a mulher grávida não ultrapasse os 200mg de consumo diário de cafeína (NE 2) (Quadro III).

Na revisão sistemática com meta-análise de Lassi e colaboradores, de 2014, foram incluídos 39 estudos, dos quais apenas nove se referiam ao consumo de cafeína no período preconcecional. Foi feita a avaliação do risco com a estratificação do consumo de cafeína inferior a $150 \mathrm{mg} /$ dia; maior a $300 \mathrm{mg} / \mathrm{dia}, 420 \mathrm{mg} / \mathrm{dia}$ e $900 \mathrm{mg} /$ dia, estimativa feita através da quantidade de bebida aferida através de questionários.

Demonstrou-se que o consumo de cafeína superior a $300 \mathrm{mg} /$ dia durante a preconceção aumentou o risco de nado morto (RR 1,31; $p=0,005$; IC 95\% 1,081,58 ), elevando-se o risco com o aumento do consumo (superior a

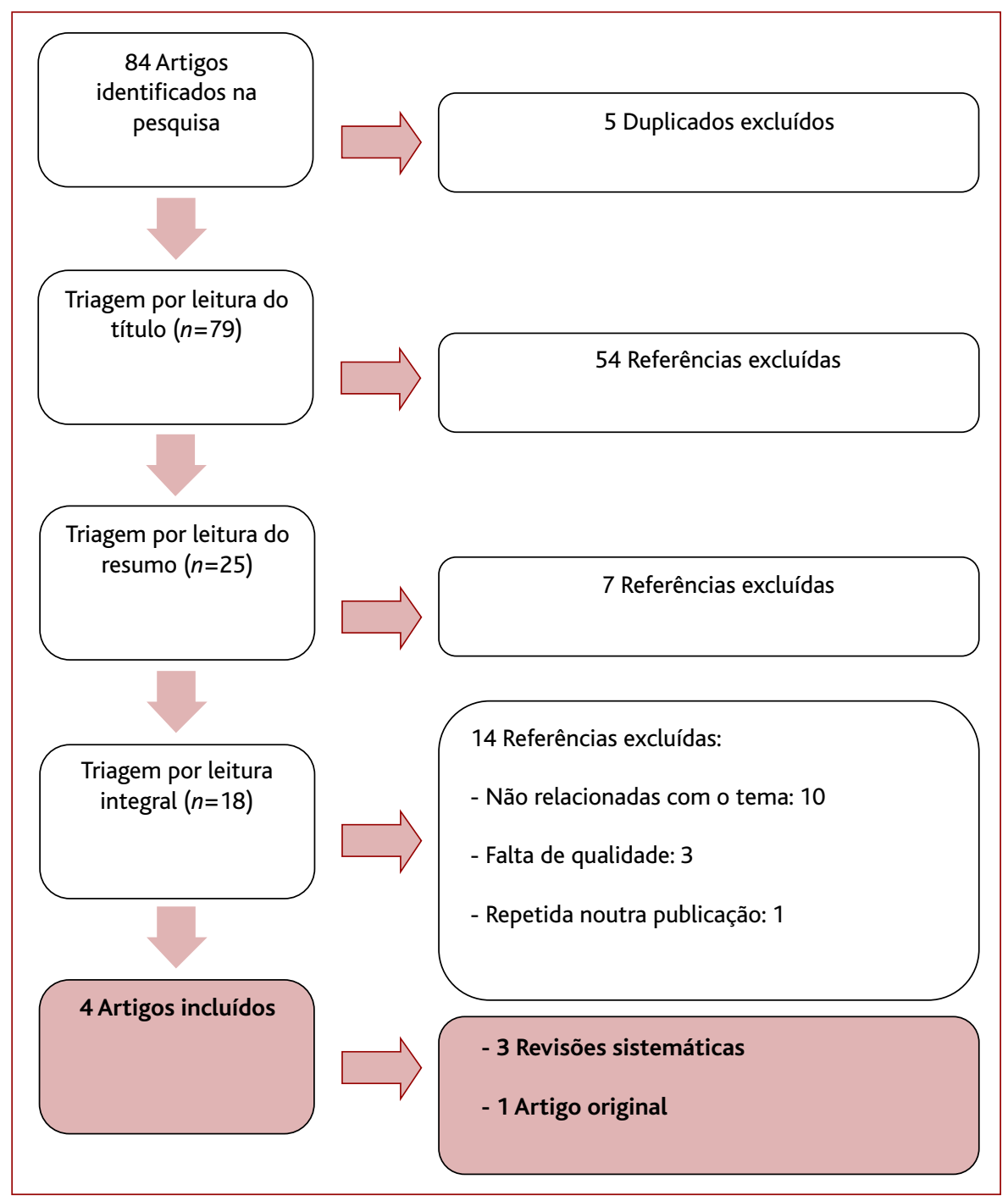

Figura 1. Fluxograma de seleção dos artigos. 420mg/dia de cafeína: RR 6,11; $p<0,0001$; IC 95\% 5,12-7,29). Não houve evidência estatisticamente significativa do aumento do risco com consumos superiores a 900mg/dia (RR 1,72; $p=0,05$; IC 95\% 1,00-2,96). Verificou-se elevada heterogeneidade entre os estudos, não tendo sido possível diminui-la devido ao reduzido número de estudos incluídos. Em relação ao abortamento espontâneo, verificou-se um aumento não significativo do risco com o consumo diário de cafeína na preconceção.

Assim, esta revisão sistemática aferiu que o consumo elevado de cafeína (superior a 300mg/dia) durante a preconceção aumenta o risco de nado morto, sendo necessária investigação adicional para averiguar a relação causal entre o consumo de cafeína e risco de abortamento espontâneo. Não obstante, é aconselhado um consumo mínimo ou nulo de cafeína durante a preconceção (NE 2) (Quadro III).

$\mathrm{O}$ estudo original de seguimento de uma coorte prospetiva, elaborado por Hahn e colaboradores em março de 2015, acompanhou 5.132 mulheres dinamarquesas, com idades entre os 18 e os 40 anos, que planeavam engravidar sem tratamento de fertilidade. Este estudo avaliou a exposição à cafeína (café ou outras bebidas) através de questionários de auto-preenchimento. Estes questionários foram preenchidos em vários momentos: na preconceção, durante a gravidez e, se aplicável, aquando da clínica de aborto espontâneo. No questionário era explicitada a quantidade de porções, em ml ou por recipiente, consumida por 


\begin{tabular}{|c|c|c|c|c|}
\hline \multicolumn{5}{|c|}{ Revisões sistemáticas } \\
\hline Referência & Estudos & Intervenção & Conclusões & NE \\
\hline $\begin{array}{l}\text { Li, et al } \\
(2015)^{10}\end{array}$ & $\begin{array}{l}26 \text { Estudos: } 13 \text { caso } \\
\text { controlo, } 13 \text { estudos } \\
\text { coorte }\end{array}$ & $\begin{array}{l}\text { Consumo de cafeína na } \\
\text { gravidez: } \\
\text { Ausência de consumo } \\
\text { vs } \\
<150 \mathrm{mg} / \text { dia } \\
\text { vs } \\
150-300 \mathrm{mg} / \text { dia } \\
\text { vs } \\
\geq 301 \mathrm{mg} / \text { dia }\end{array}$ & $\begin{array}{l}\text { O consumo de cafeína durante a gravidez associou-se a } \\
\text { aumento do risco de abortamento espontâneo e de } \\
\text { nado morto - OR } 1,47(p<0,01 \text {; IC } 95 \% \text { 1,31-1,66). } \\
\text { Encontrou-se um efeito dose-resposta para consumos } \\
\text { superiores a 149,5mg/dia, e um aumento do risco 19\% } \\
\text { para cada aumento no consumo de 150mg/dia. }\end{array}$ & 2 \\
\hline $\begin{array}{l}\text { Greenwood, } \\
\text { et al }(2014)^{12}\end{array}$ & $\begin{array}{l}53 \text { Estudos } \\
\text { observacionais de } \\
\text { interesse } \\
26 \text { Estudos } \\
\text { abortamento } \\
\text { espontâneo } \\
5 \text { Estudos nado } \\
\text { morto }\end{array}$ & $\begin{array}{l}\text { Consumo de cafeína na } \\
\text { gravidez: } \\
<100 \mathrm{mg} / \text { dia } \\
\text { vs } \\
\geq 100 \mathrm{mg} / \text { dia }\end{array}$ & $\begin{array}{l}\text { Existe uma associação entre o consumo de cafeína } \\
\text { maior que } 100 \mathrm{mg} / \text { dia durante a gravidez e risco de } \\
\text { abortamento espontâneo - RR } 1,11(p<0,001 ; \text { IC } 95 \% \\
\text { 1,07-1,14) e de nado morto - RR } 1,19(p=0,007 ; \\
\text { IC } 95 \% 1,05-1,35) \text {. }\end{array}$ & 2 \\
\hline $\begin{array}{l}\text { Lassi, et al } \\
(2014)^{19}\end{array}$ & $\begin{array}{l}39 \text { Estudos } \\
\text { observacionais - de } \\
\text { interesse } 9 \text { estudos }\end{array}$ & $\begin{array}{l}\text { Consumo de cafeína } \\
\text { na preconceção: } \\
<150 \mathrm{mg} / \text { dia } \\
\text { vs } \\
>300 \mathrm{mg} / \mathrm{dia} \\
\text { vs } \\
>420 \mathrm{mg} / \text { dia } \\
\text { vs } \\
>900 \mathrm{mg} / \text { dia }\end{array}$ & $\begin{array}{l}\text { O consumo elevado de cafeína ( }>300 \mathrm{mg} / \text { dia) durante a } \\
\text { preconceção aumenta o risco de nado morto - RR 1,31 } \\
\text { ( } p=0,005 ; \text { IC } 95 \% 1,08-1,58 \text { ), não havendo evidência } \\
\text { estatisticamente significativa relativamente ao } \\
\text { abortamento espontâneo. }\end{array}$ & 2 \\
\hline
\end{tabular}

Legenda: IC - Intervalo de confiança; NE - Nível de evidência; OR - Odds ratio; RR - Risco relativo.

semana de: café (250ml, caneca), café descafeínado (250ml, caneca), ervas para infusão/verde (250ml, caneca), chá preto $(250 \mathrm{ml}$, caneca), cola normal (500ml, garrafa) e cola light (500ml, garrafa). Foram consideradas as seguintes doses de cafeína em mg, cada dose: café $141 \mathrm{mg}$, café descafeínado $5 \mathrm{mg}$, chá preto $56 \mathrm{mg}$, cola normal $51 \mathrm{mg}$ e cola light $60 \mathrm{mg}$. Avaliaram-se potenciais variáveis confundidoras utilizando regressão de Cox - idade materna, paridade, tabagismo, história de abortos espontâneos, consumo de álcool, atividade física, peso e altura e habilitações literárias.

Registou-se um follow up adequado, com um seguimento de $87 \%$ da população. Os resultados mostraram que, na população, uma amostra de 732 mulheres teve um aborto espontâneo. No período preconceção, o consumo de cafeína não foi associado a um risco substancial de aborto espontâ- neo. O hazard ratios (HR) de consumo de cafeína $\geq 300 \mathrm{mg} /$ dia foi de 1,09 (IC 95\% 0,89-1,33) em comparação com 100mg/dia. Consumos no início da gravidez de 100199, 200-299 e maior ou igual a 300mg de cafeína/dia mostraram HR de 1,62 (IC 95\% 1,19-2,22), 1,48 (IC 95\% 1,03-2,13) e 1,23 (IC 95\% 0,61-2,46), respetivamente, comparativamente a consumos $<100 \mathrm{mg} /$ dia.

Nesta coorte prospetiva conclui-se que o consumo de cafeína, principalmente durante a gravidez, foi associado a um risco ligeiramente maior de aborto espontâneo, pelo que o consumo deve ser desaconselhado, ainda que a evidência da relação dose-resposta demonstrada tenha sido reduzida.

Como limitações, os autores deste estudo original apontaram a ausência da colheita diária de hormona gonadotrófica coriónica humana, com prejuízo da taxa de deteção de 


\section{QUADRO IV. Estudos originais}

Estudo coorte prospetivo

\begin{tabular}{|c|c|c|c|c|}
\hline Referência & População & Intervenção & Conclusões & NE \\
\hline $\begin{array}{l}\text { Hahn, et al } \\
(2015)^{20}\end{array}$ & 5.132 Mulheres & $\begin{array}{l}\text { Consumo de cafeína na } \\
\text { gravidez e preconceção: } \\
<100 \mathrm{mg} / \text { dia } \\
\text { vs } \\
100-199 \mathrm{mg} / \text { dia } \\
\text { vs } \\
200-299 \mathrm{mg} / \text { dia } \\
\text { vs } \\
\geq 300 \mathrm{mg} / \text { dia }\end{array}$ & $\begin{array}{l}\text { O consumo de cafeína na preconceção não foi associado a um } \\
\text { risco substancial de aborto espontâneo. } \\
\text { O consumo de cafeína durante a gravidez foi associado a um } \\
\text { risco ligeiramente maior de aborto espontâneo, mas houve } \\
\text { pouca evidência na relação dose-resposta. }\end{array}$ & 1 \\
\hline
\end{tabular}

Legenda: NE - Nível de evidência.

abortos espontâneos; o viés de fiabilidade da informação fornecida pela mulher (relativa aos seus consumos) e a possível variação individual no metabolismo da cafeína, devido a polimorfismos genéticos, que afete a quantidade de cafeína em circulação, a qual não foi estudada (NE 1) (Quadro IV).

\section{CONCLUSÃO}

O consumo diário de cafeína moderado a elevado durante a gestação parece estar associado a um aumento do risco de abortamento espontâneo ou nado morto, propondo-se ingestões inferiores a 100-150mg/dia nesta fase (força de recomendação B). Relativamente ao consumo durante a preconceção não há ainda evidência suficiente que demonstre associação entre o consumo de cafeína e morte in utero.

Os autores ressalvam a inexistência de normas de orientação clínica robustas acerca deste tema e a existência apenas de estudos de natureza observacional, dadas as limitações éticas que restringem a experimentação no período gravídico. De salientar que este tipo de estudos poderá estar associado a um possível viés de memória. A utilização de diferentes definições temporais de abortamento espontâneo e nado morto, não correspondentes às aceites na legislação portuguesa, constituiu uma limitação importante. Outra limitação prende-se com a real quantificação do consumo diário de cafeína-quer pela inclusão de diferentes fontes de cafeína na alimentação, divergente entre estudos; quer devido à utilização de diferentes tabelas de estimativa de quantidade de cafeína por quantidade/porção de alimento, potenciada pela elevada variabilidade intercultural da quantidade de cafeína, conforme o tipo de café e a forma de preparação; quer devido à variabilidade nos intervalos de consumo definidos nos diferentes trabalhos. $\mathrm{O}$ ajuste para potenciais variáveis confundidoras, como o consumo tabágico e a idade materna, nem sempre foi realizado. A redução do consumo de cafeína na presença de sintomas associados a um pregnancy signal elevado não foi despistada. Também não foram estudadas variações individuais do metabolismo da cafeína devidas a polimorfismos genéticos.

A importância desta temática na prática clínica fundamenta a realização de estudos mais abrangentes, com ajuste metodológico adequado para as variáveis confundidoras identificadas e assegurando a quantificação precisa dos consumos de cafeína na população em estudo.

Não obstante, a presente revisão baseada na evidência recomenda que as mulheres grávidas, ou que ponderam engravidar, devem restringir ao mínimo o seu consumo diário de cafeína. Dado o café ser o produto com cafeína mais comummente consumido na nossa cultura, e tendo em conta a elevada concentração de cafeína no café, aconselham-se as mulheres grávidas ou que ponderem engravidar a restringir o consumo de café a uma chávena por dia.

Deve-se ainda alertar as mulheres para a presença de cafeína noutros produtos, sugerindo uma leitura atenta dos seus rótulos.

\section{REFERÊNCIAS BIBLIOGRÁFICAS}

1. Patel S, Rizzolo D. Q: is caffeine safe during pregnancy? J Am Acad Physician Assist. 2012;25(5):69.

2. European Coffee Federation. European coffee report 2013/14: European chapter and key national data [Internet]. Brussels: ECF; 2014. Available from: http://www.stc-coffee.com/wp-content/uploads/2014/08/European_Coffee_Report_2013-14_European_chapter.pdf

3. Oliveira T, Dias RR. Perspectivas e tendências sociais no consumo de café em Portugal para 2021. In: Dias RR, Afonso JC, editors. Marketing agroalimentar: fundamentos e estudos de caso. Lisboa: Vida Económica; 2015. p. 243-54. ISBN 9789897680892

4. Kirkinen P, Jouppila P, Koivula A, Vuori J, Puukka M. The effect of caffeine on pla- 
cental and fetal blood flow in human pregnancy. Am J Obstet Gynecol. 1983; 147(8):939-42.

5. CARE Study Group. Maternal caffeine intake during pregnancy and risk of fetal growth restriction: a large prospective observational study. BMJ.2008;337:a2332.

6. Grosso LM, Rosenberg KD, Belanger K, Saftlas AF, Leaderer B, Bracken MB. Maternal caffeine intake and intrauterine growth retardation. Epidemiology 2001;12(4):447-55.

7. Soyka LF. Effects of methylxanthines on the fetus. Clin Perinatol. 1979;6(1):3751.

8. Administração Central do Sistema de Saúde. As semanas de gestação na codificação da obstetrícia [Internet]. Lisboa:ACSS; 2011 [cited 2015 Sep 1]. Available from: http://portalcodgdh.min-saude.pt/index.php/As_semanas_de_gesta\% C3\%A7\%C3\%A3o_na_codifica\%C3\%A7\%C3\%A3o_da_obstetr\%C3\%ADcia

9. Ouyang F, Zhang J, Betrán AP, Yang Z, Souza JP, Merialdi M. Recurrence of adverse perinatal outcomes in developing countries. Bull World Health Organ. 2013;91(5):357-67.

10. Li J, Zhao H, Song JM, Zhang J, Tang YL, Xin CM. A meta-analysis of risk of pregnancy loss and caffeine and coffee consumption during pregnancy. Int J Gynaecol Obstet. 2015;130(2):116-22.

11. Peck JD, Leviton A, Cowan LD. A review of the epidemiologic evidence concerning the reproductive health effects of caffeine consumption: a 2000-2009 update. Food Chem Toxicol. 2010;48(10):2549-76.

12. Greenwood DC, Thatcher NJ, Ye J, Garrard L, Keogh G, King LG, et al. Caffeine intake during pregnancy and adverse birth outcomes: a systematic review and dose-response meta-analysis. Eur J Epidemiol. 2014;29(10):725-34

13. Graça LM. Medicina materno-fetal. 4a ed. Lisboa: LIDEL; 2010. ISBN 9789727 576548

14. National Institute for Health and Care Excellence. Antenatal care for uncomplicated pregnancies (CG62) [Internet]. London: NICE; 2008 [updated 2017 Jan]. Available from: https://www.nice.org.uk/guidance/cg62

15. Ebell MH, Siwek J, Weiss BD, Woolf SH, Susman J, Ewingman B, et al. Strength of recommendation taxonomy (SORT): a patient-centered approach to grading evidence in the medical literature. Am Fam Physician. 2004;69(3):548-56.
16. Downs SH, Black N. The feasibility of creating a checklist for the assessment of the methodological quality both of randomised and non-randomised studies of health care interventions. J Epidemiol Community Health. 1998;52(6):377-84.

17. AGREE Collaborations. Development and validation of an international appraisa instrument for assessing the quality of clinical practice guidelines: the AGREE project. Qual Saf Health Care. 2003;12(1):18-23.

18. Brouwers M, Kho ME, Browman GP, Cluzeau F, Feder G, Fervers B, et al. AGREE II: advancing guideline development, reporting and evaluation in healthcare [Internet]. The AGREE Research Trust; 2009 [cited 2009 Sep 1] Available from: http://www.agreetrust.org/wp-content/uploads/2013/10/AGREE-II-Users-Manual-and-23-item-Instrument_2009_UPDATE_2013.pdf

19. Lassi ZS, Imam AM, Dean SV, Bhutta ZA. Preconception care: caffeine, smoking, alcohol, drugs and other environmental chemical/radiation exposure. Reprod Health. 2014;11 Suppl 3:S6.

20. Hahn KA, Wise LA, Rothman KJ, Mikkelsen EM, Brogly SB, Sørensen HT, et al. Caffeine and caffeinated beverage consumption and risk of spontaneous abortion. Hum Reprod. 2015;30(5):1246-55.

21. Mitchell DC, Knight CA, Hockenberry J, Teplansky R, Hartman TJ. Beverage caffeine intake in the U.S. Food Chem Toxicol. 2014;63:136-42.

22. Harland BF. Caffeine and nutrition. Nutrition. 2000;16(7-8):522-6.

23. McCusker RR, Goldberger BA, Cone EJ. Caffeine content of specialty coffees. JAnal Toxicol. 2003;27(7):520-2

\section{CONFLITOS DE INTERESSE}

As autoras declaram não ter conflitos de interesse.

\section{ENDEREÇO PARA CORRESPONDÊNCIA}

Filipa Alexandra Lopes Matias

R. Calouste Gulbenkian, $n^{\circ}$ 237-1H1, 4465-040 Porto

E-mail: filipamatias.mgf@gmail.com

Recebido em 13-02-2016

Aceite para publicação em 16-11-2016

\section{ABSTRACT}

\section{CAFFEINE CONSUMPTION: WHAT TO RECOMMEND DURING PRECONCEPTION AND PREGNANCY?}

Objective: To determine if there is a relationship between caffeine consumption during preconception and pregnancy and the occurrence of spontaneous abortion or stillbirth in healthy women.

Data sources: MEDLINE database, National Guideline Clearinghouse, Canadian Medical Association Practice Guidelines Infobase, Cochrane Library, Bandolier, DARE, Trip Database, and Index of Portuguese medical journals.

Methods: An evidence based review of relevant scientific papers published within the past five years (from 01/08/2010 to 31/08/2015), in English, French, Italian, Spanish and Portuguese was conducted. The MESH terms used were 'caffeine', 'spontaneous abortion' and 'fetal death'. To evaluate the levels of evidence (LE) and strength of recommendation, the authors used the Strength of recommendation taxonomy of the American Family Physician.

Results: The search revealed 84 papers, four of which were included in this survey. We included three systematic reviews (all with LE 2) and one prospective cohort study (LE 1). These papers show an increased occurrence of spontaneous abortion or stillbirth with moderate-to-high consumption of caffeine (over 100-150 mg/day) during pregnancy. There is insufficient evidence for an association between these adverse events and caffeine consumption during preconception.

Conclusions: Moderate to high caffeine consumption during pregnancy increases the risk of spontaneous abortion and stillbirth (Strength of recommendation B). We recommend that caffeine consumption during this period should be reduced $(<100-150 \mathrm{mg} /$ day) or completely avoided. There is insufficient evidence for recommendations regarding caffeine consumption during preconception. Better quality studies are needed to confirm the available evidence.

Keywords: Caffeine; Spontaneous abortion; Fetal death; Stillbirth; Pregnancy; Preconception 